\title{
Kinetic study of the photodecoloration mechanism of an inversely photochromic class of compounds forming spiropyran analogues ${ }^{\dagger}$
}

\author{
D. Kühn a, H. Balli ${ }^{\mathrm{b}}$ and U. E. Steiner ${ }^{\mathrm{a}, \boldsymbol{\dagger}}$ \\ "Fakultät für Chemie, Universität Konstanz, W-7750 Konstanz (FRG) \\ 'Institut für Farbenchemie, Universität Basel, St.-Johanns-Vorstadt 10/12, CH-4056 Basel \\ (Switzerland)
}

\begin{abstract}
The quantum yields and kinetics of the photodecoloration of five representatives of an inversely photochromic class of compounds, reacting to give spiro compounds related to the indolinospiropyrans by replacement of the chromene part of the molecule by an imidazodihydroquinoline fragment, were investigated by stationary illumination and nanosecond time-resolved laser flash spectroscopy. In particular, the role of the ground state of the open-chain cis conformer as an intermediate in spiro-bond formation (photodecoloration) and opening (thermocoloration) was quantitatively assessed.
\end{abstract}

\section{Introduction}

Photochromic compounds and their molecular transformations have attracted the interest of photochemists for many years [1-3] and are the subject of a recent comprehensive monograph [4]. The interest in these compounds is strongly focused on their intriguing potential as materials for optical data storage and processing, but the mechanisms of their transformations also continue to provide interesting problems for basic scientific research.

One class of photochromic compounds which has figured most prominently in this field is the so-called spiropyrans ( $c f$. ref. 5) with thcir prototype compound 1,3,3trimethylindolinospirobenzopyran (SP) (Scheme 1).

Here the spiropyran SP is a colourless compound which is photochemically transformed by UV absorption into an intensely coloured open-chain merocyanine chromophore $M$. The reaction is thermoreversible, but the rate of thermal decoloration $(\mathrm{M} \rightarrow \mathrm{SP}$ ) may vary greatly, depending on the particular derivative $[5,6]$. While the merocyanine form is most stable in the extended trans conformation, photochemical opening of the spiro bond or thermal ring closure will necessarily involve the passing of the system through a cisoid conformation denoted as $X$ in Scheme 1. The identification of this conformer has attracted the interest of research workers [2,7]. There is now ample evidence for its detectability [8-11] in highly viscous media at low temperature.

\footnotetext{
'Dedicated to Professor Schaffner on the occasion of his 60th birthday.

${ }^{\dagger \dagger}$ Author to whom correspondence should be addressed.
} 
<smiles>CN1c2ccccc2C(C)(C)C1(C)Oc1ccc2ccccc2c1</smiles>

SP

Scheme 1 .
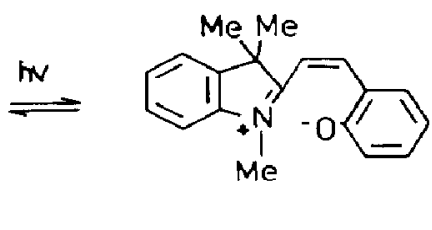

$\mathbf{X}$<smiles>CC1(C)c2ccccc2[N+]1(C)C</smiles>

$M$

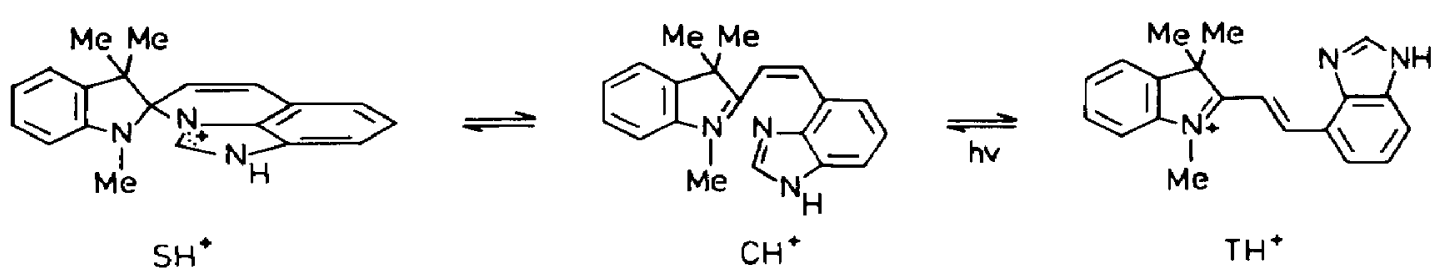

Scheme 2.

Several years ago a variation of the spiropyran structure was developed by the research group of one of us (H. B.) at Basel $[12,13]$. This variation was devised to yield inversely photochromic compounds, i.e. structures that are reversibly photobleached and undergo thermal coloration. The principle of these compounds is shown in Scheme 2.

The chromene chromophore of the spiropyran is replaced by a dihydroquinoline unit with its spiro-C-bound nitrogen atom further incorporated into another anellated imidazole ring. Thus, by protonation of the spiro form $S$, the imidazole-N-spiro-C bond becomes labile and a proton-induced thermal coloration occurs due to the formation of the open-chain conjugated chromophore $\mathrm{TH}^{+}$. Photochemically, a reversible decoloration of $\mathrm{TH}^{+}$is observed whereby the form $\mathrm{SH}^{+}$is regenerated. It has been suggested $[12,13]$ that thermocoloration of $\mathrm{SH}^{+}$and photodecoloration of $\mathrm{TH}^{+}$both $^{-}$ involve the ground state of the open-chain cis isomer $\mathrm{CH}^{+}$. So far, however, no quantitative evidence has been provided.

In this paper, we present a detailed study by stationary and time-resolved kinetic measurements of the role of the open-chain cis conformer in thermocoloration and photodecoloration of some compounds of type $S$.

\section{Experimental details}

\subsection{Materials}

The inversely photochromic compounds were synthesized at the Institut für Farbenchemie, Basel, by A. Keller (1 and 2 [14]) and R. Konstanzer (3-5 [15]). Compound 1 was available as a monovalent salt $1 \mathrm{TEt}^{+} \mathrm{BF}_{4}^{-}, 3$ and 4 as divalent salts $\mathrm{TH}_{2}{ }^{2+}\left(\mathrm{ClO}_{4}{ }^{-}\right)_{2}$ and 2 and 5 as neutral spiro compounds.

The solvent used was methanol (Merck, p.A.). For acidification, suitable amounts of $5 \mathrm{n}$ aqueous $\mathrm{HCl}$ (Riedel de Haen, Fixanal) were added. 


\subsection{Instrumentation}

Absorption spectra were recorded on a Perkin-Elmer Lambda 5 UV-visible spectrophotometer. For continuous irradiation experiments, a mercury high pressure light source (Osram HBO $200 \mathrm{~W} / 2$ ), combined with a $436 \mathrm{~nm}$ interference filter, was used. The solutions were irradiated in $1 \mathrm{~cm}$ fluorescence cuvettes, equipped with magnetic stirring and súrrounded by a temperature-controlled support. For actinometry, the azobenzene cis-trans photoisomerization reaction was applied following the method of Gauglitz and Hubig [16].

The equipment used for laser flash photolysis is described in detail in refs. 17 and 18. The photolysing laser source was an excimer-laser-pumped dye laser of about $20 \mathrm{~ns}$ pulse width and 4-6 $\mathrm{mJ}$ pulse energy operated with the laser dyes Lambdachrome 4200 Stilben $3(410-480 \mathrm{~nm})$ or Lambdachrome 5000 Coumarin $307(480-550 \mathrm{~nm})$. Transient absorption was measured using a pulsed probe light from a high pressure xenon lamp (Osram XBO 150) and recorded on a Tektronix 7912 AD digitizer. For measuring temperature-dependent kinetics, the sample cuvette was housed in a temperature-controllable optical cryostat (Oxford $\mathrm{LN}_{2}$ ) allowing for heating and cooling to adjust temperatures between liquid nitrogen temperature and $230^{\circ} \mathrm{C}$. In the present study, a temperature range of between -25 and $50^{\circ} \mathrm{C}$ was used for determining the activation parameters of the ring-closure reaction.

\section{Results and discussion}

The following compounds with their spiro forms, denoted as $1 S E t^{+}, 2 S, 3 S, 4 S$ and $5 \mathrm{~S}$, were investigated.

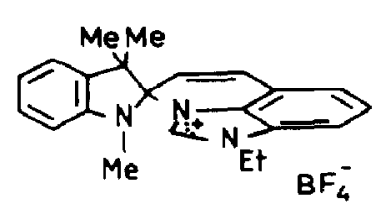

1SE+ +

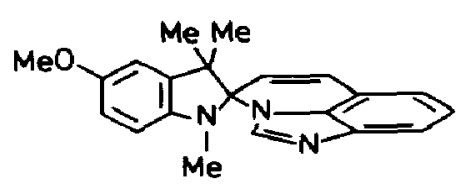

35

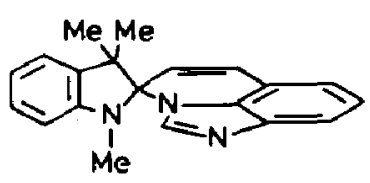

$2 S$

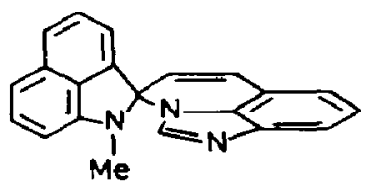

$4 S$

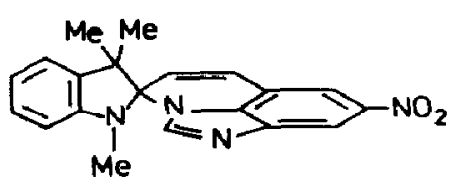

55

While $2 S$ represents the basic structure, the effect of chemical variation in the indolino or dihydroquinoline part can be studied using compounds 3S-5S. As mentioned in Section 1, thermal ring opening (coloration) requires protonation at the exposed 


$$
\begin{aligned}
& \mathrm{TH}_{2}^{++} \frac{\phi_{\mathrm{tc}}^{*}}{\mathrm{k}_{\mathrm{ct}}^{*}} \mathrm{CH}_{2}^{++} \\
& K_{0}^{\top}\|\quad\| K_{a}^{c}
\end{aligned}
$$

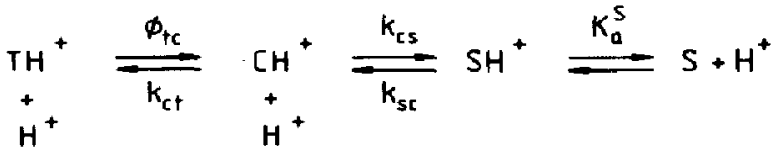

Scheme 3.

imidazole nitrogen atom. In acidified solutions, the overall system of reactions to be considered is as represented in Scheme 3 , which also shows the notation of the various rate and equilibrium constants. It can be seen that acidity of environment after thermal ring opening of $\mathrm{SH}^{+}$leading, via the potentially detectable open-chain conformer $\mathrm{CH}^{+}$, to the thermodynamically stable $\mathrm{TH}^{+}$, a second stage of protonation affording the species $\mathrm{CH}_{2}{ }^{2+}$ and $\mathrm{TH}_{2}{ }^{2+}$ eventually has to be taken into account. Since, in principle, this may cause some complications in the kinetic analysis if $\mathrm{p} K_{\mathrm{a}}^{\mathrm{S}}, \mathrm{p} K_{\mathrm{a}}^{\mathrm{C}}$ and $\mathrm{p} K_{\mathrm{a}}^{\mathrm{T}}$ are not clearly separated, we found it convenient to start our investigation with the ethylated cation 1SEt ${ }^{+}$, where it is not necessary to work in acidified solutions and no protolytic complications are expected.

\subsection{Stationary experiments with $1 S E t^{+}$and $1 T E t^{+}$}

As noted by Keller [14], solutions of the coloured salt $1 \mathrm{TEt}^{+} \mathrm{BF}_{4}{ }^{-}$undergo efficient photobleaching whereby the spiro form $\mathrm{ISEt}^{+}$is formed. We measured this reaction quantitatively using irradiation with the mercury line at $436 \mathrm{~nm}$. A representation of the spectral change during this photoreaction is shown in Fig. 1. The long-wavelength absorption of $\mathrm{TEt}^{+}$disappears almost completely, while the typical absorption band of the spiro form SEt ${ }^{+}$with a maximum at about $280 \mathrm{~nm}$ is developed. The chemical change must correspond to a uniform reaction as follows from the perfectly linear $E / E$ diagram (see Fig. 1(b)). The uniformity of the reaction does not necessarily mean that only two chemical species $\left(\mathrm{TEt}^{+}\right.$and $\left.\mathrm{SEt}^{+}\right)$are present; a certain stationary concentration of $\mathrm{CEt}^{+}$may be present but in a fast equilibrium with $\mathrm{SEt}^{+}$. After the end of the reaction, a weak absorbance tail remains in the region around $400 \mathrm{~nm}$ which cannot be due to $\mathrm{SEt}^{+}$but may be attributed to some small equilibrium concentration of $\mathrm{CEt}^{+}$. For a quantitative determination of the quantum yield $\phi\left(\mathrm{TEt}^{+} \rightarrow \mathrm{SEt}^{+}\right)$, the weak $\mathrm{CEt}^{+}$absorption at $436 \mathrm{~nm}$ can be neglected and the photokinetic curve may be analysed according to the relation [19] (see Fig. 2)

$\log \frac{10^{E t}-1}{10^{E r(0)}-1}=\phi\left(\mathrm{TEt}^{+} \longrightarrow \mathrm{SEt}^{+}\right) \epsilon_{\mathrm{TE} t} I_{0} \mathrm{~d} t$

where $E^{\prime}$ is the absorbance at $436 \mathrm{~nm}$, and the meaning of the other quantities is obvious. The quantum yield obtained is $\phi\left(\mathrm{TEt}^{+} \rightarrow \mathrm{SEt}^{+}\right)=0.54 \pm 0.05$. If it is assumed that the photochemical step proper is a trans-cis photoisomerization, the effective value of $\phi\left(\mathrm{TEt}^{+} \rightarrow \mathrm{SEt}{ }^{+}\right)$corresponds to

$\phi\left(\mathrm{TEt}^{+} \longrightarrow \mathrm{SEt}{ }^{+}\right)=\phi_{\mathrm{tc}} \frac{k_{\mathrm{cs}}}{k_{\mathrm{cs}}+k_{\mathrm{ct}}}$ 

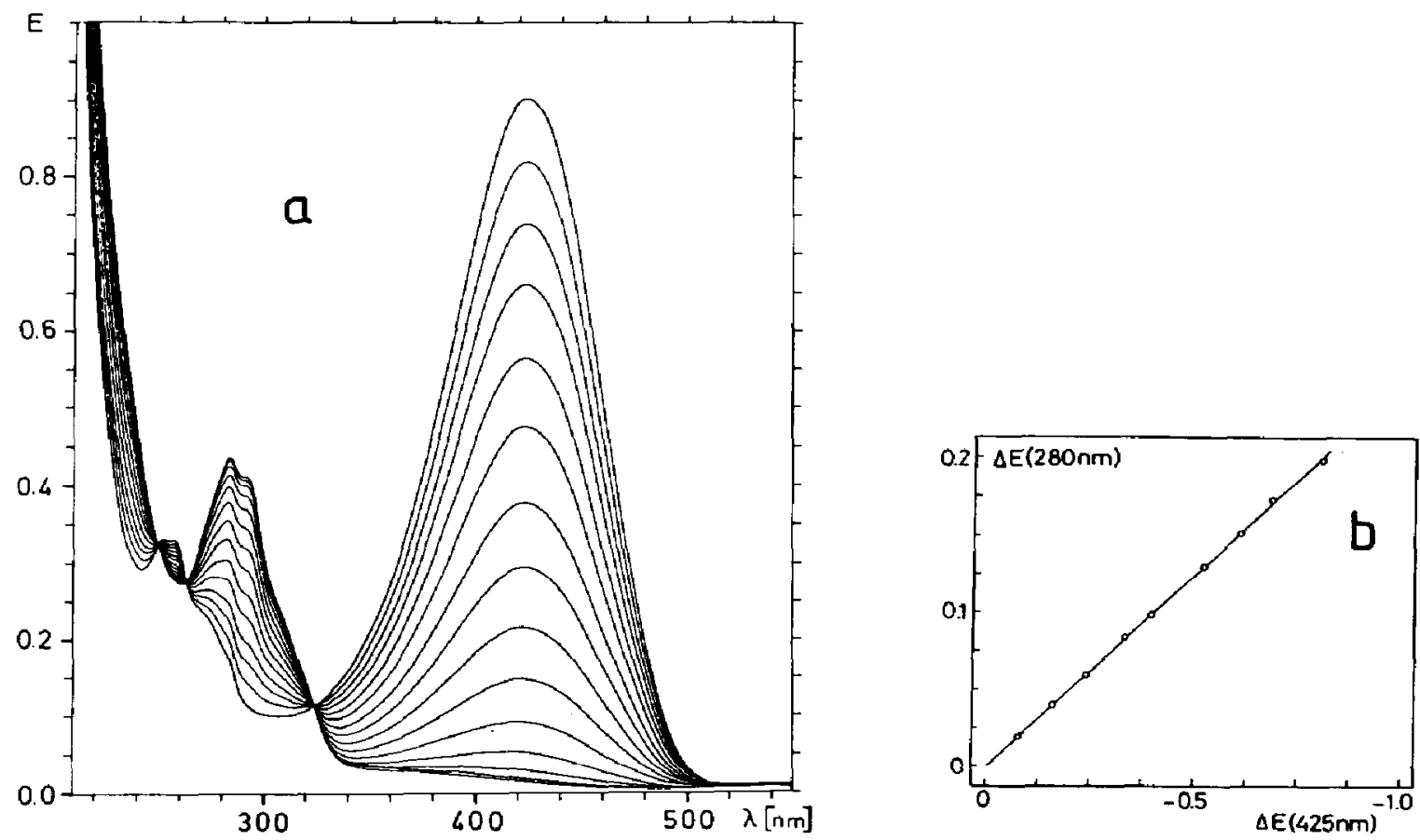

Fig. 1. (a) Absorption spectra recorded during irradiation at $436 \mathrm{~nm}$ of $1 \mathrm{TEt}^{+}\left(c=2.75 \times 10^{-5}\right.$ $M$ ) in methanol. (b) Demonstration of linear correlation of absorbance changes $\Delta E$ at two wavelengths.

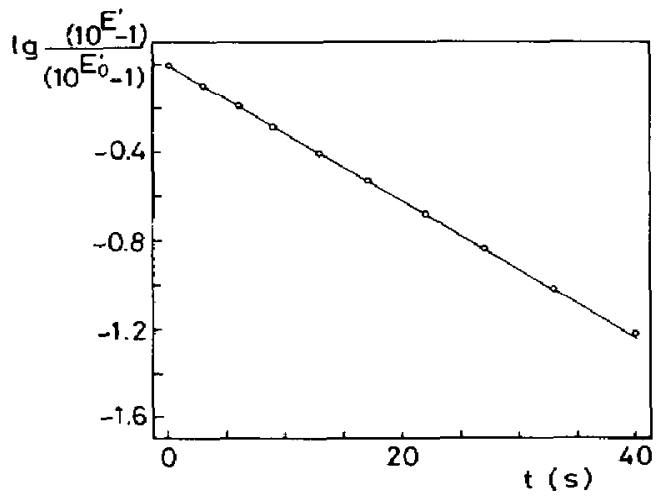

Fig. 2. Plot for determination of photobleaching quantum yield of $1 \mathrm{TE}^{+}{ }^{+}$according to eqn. (1) (photon irradiance at $436 \mathrm{~nm}, I_{0}=1.84 \times 10^{-9}$ einsteins $\mathrm{s}^{-1} \mathrm{~cm}^{-2}$; extinction coefficient of 1TEt ${ }^{+}$ at $436 \mathrm{~nm}, 31100 \mathrm{M}^{-1} \mathrm{~cm}^{-1}$ ).

The value found for $\phi\left(\mathrm{TEt}^{+} \rightarrow \mathrm{SEt}^{+}\right)$is of an order of magnitude typical for photochemical trans-cis isomerizations (e.g. stilbene, 0.50 [20]; 4-styrylpyridinium cation, 0.53 [21]) and may directly correspond to the quantum yield $\phi_{\mathrm{tc}}$. In this case, $k_{\mathrm{cs}} \gg k_{\mathrm{ct}}$, which is demonstrated below.

In the dark, the spiro form $1 \mathrm{SEt}^{+}$reverts to the open form $1 \mathrm{TEt}^{+}$with a rate constant of $0.03 \mathrm{~h}^{-1}$ (room temperature; solvent, methanol). In terms of the involvement of the open form $1 \mathrm{CEt}^{+}$, this rate constant may be expressed as 
$k\left(\mathrm{SEt}^{+} \longrightarrow \mathrm{TEt}^{+}\right)=k_{\mathrm{ct}} k_{\mathrm{sc}} / k_{\mathrm{cs}}=3.5 \times 10^{-4} \mathrm{~s}^{-1}$

From the extinction coefficient of $1 \mathrm{CEt}^{+}$determined below and the weak long-wavelength absorbance in equilibrium with $1 \mathrm{SEt}^{+}$, the equilibrium constant may be estimated as

$K_{\mathrm{c} / \mathrm{s}}=k_{\mathrm{sc}} / k_{\mathrm{cs}} \approx 0.07$

leading to $k_{\mathrm{ct}} \approx 5 \times 10^{-3} \mathrm{~s}^{-1}$.

In order to confirm the reaction scheme (see Scheme 3), it is instructive to investigate the behaviour of the dicationic forms $\mathrm{TEtH}^{2+}$ and $\mathrm{CEtH}^{2+}$. The $\mathrm{pK} K_{\mathrm{a}}$ value of the reaction

$1 \mathrm{TEtH}^{2+} \rightleftarrows 1 \mathrm{TEt}^{+}+\mathrm{H}^{+}$

is 2.6. During irradiation of solutions of $1 \mathrm{TEtH}^{2+}$ (see Fig. 3), a uniform photoconversion takes place, yielding a spectrum which clearly indicates the conservation of the fully conjugated $\pi$ system. Obviously, the photoreaction ends with the dicationic conformer $1 \mathrm{CEtH}{ }^{2+}$ [14]. The spectral change, blue shift and decrease in the intensity of the long-wavelength absorption band, is similar to that observed with stilbene and hence consistent with this interpretation. The quantum yield of this photoreaction for an irradiation wavelength of $436 \mathrm{~nm}$ is $\phi_{\mathrm{tc}}^{\prime}=0.57 \pm 0.05$. This is similar to the value of $\phi_{\mathrm{tc}}$ for the monocation $1 \mathrm{TEt}^{+}$.

Whereas the spiropyran $1 \mathrm{SEt}^{+}$is not formed from $1 \mathrm{CEtH}^{2+}$, it is possible to study the thermal formation of $1 \mathrm{CEtH}^{2+}$ when starting with $1 \mathrm{SEt}^{+}$in acidified solutions (see Fig. 4). For the overall process

$1 \mathrm{SEt}{ }^{+} \rightleftarrows 1 \mathrm{CEt}^{+} \rightleftarrows 1 \mathrm{CEtH}^{2+}$

a value of 1.1 is found for $p K_{\text {tot }}$, defined as

$\mathrm{p} K_{\mathrm{tot}}=-\log \frac{\left[\mathrm{SEt}^{+}\right]\left[\mathrm{H}^{+}\right]}{\left[\mathrm{CEtH}^{2+}\right]}$

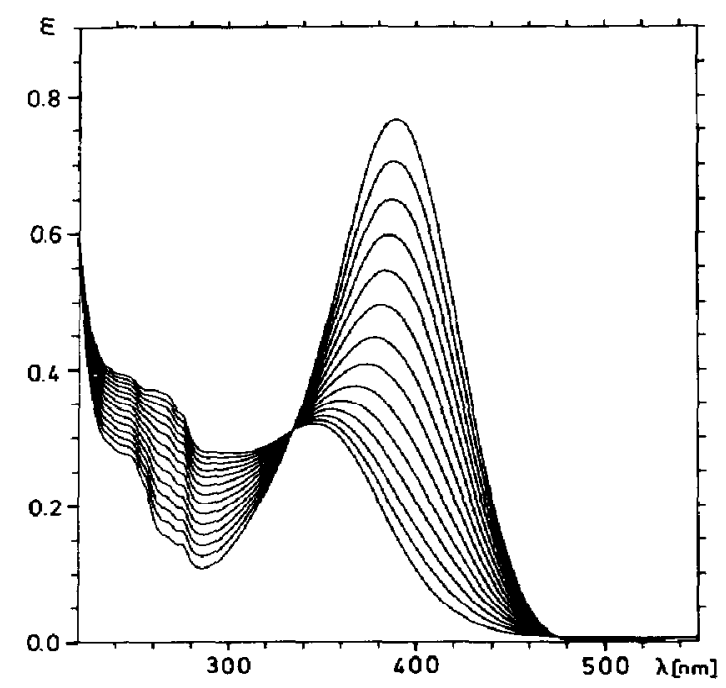

Fig. 3. Absorption spectra recorded during irradiation at $436 \mathrm{~nm}$ of $1 \mathrm{TEtH}^{2+}\left(c=2.75 \times 10^{-5}\right.$ M) in methanol. 

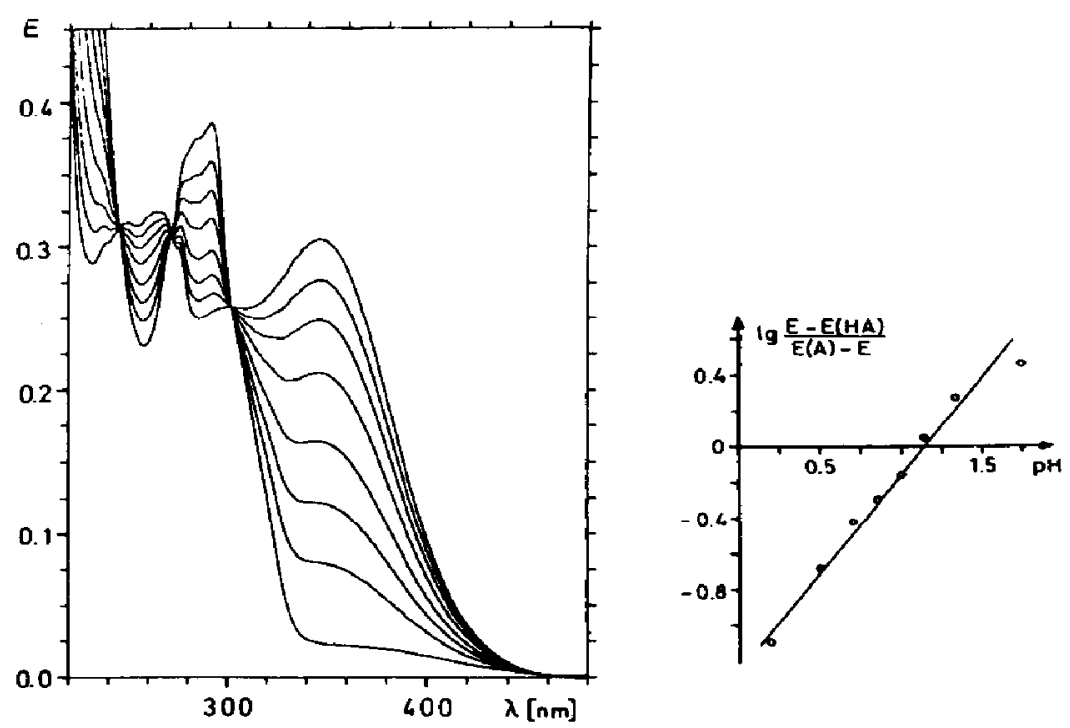

Fig. 4. Spectral absorbance change due to a shift in the equilibrium $1 \mathrm{SEt}^{+}-\mathrm{ICEtH}^{2+}$ with $\mathrm{pH}$ and determination of $\mathrm{p} K_{\mathrm{a}}$ in methanol. The dye concentration was $2.75 \times 10^{-5} \mathrm{M}$.

The form $1 \mathrm{CEtH}^{2+}$ thus produced, transforms to $1 \mathrm{TEtH}^{2+}$ only very slowly with a rate constant of $k_{\mathrm{ct}}^{\prime}=3.4 \times 10^{-4} \mathrm{~s}^{-1}$. As in the photochemical case $\left(\phi_{\mathrm{tc}} \approx \phi_{\mathrm{tc}}^{\prime}\right)$, this result is again very similar to that for the monocationic form (i.e. $k_{\mathrm{ct}} \approx k_{\mathrm{ct}}^{\prime}$ ).

Using $p K_{\text {tor }}$ we can write for $\mathrm{p} K_{\mathrm{n}}^{\mathrm{C}}$

$\mathrm{p} K_{\mathrm{a}}^{\mathrm{C}}=\mathrm{p} K_{\mathrm{tot}}+\log \left(k_{\mathrm{cs}} / k_{\mathrm{sc}}\right)$

which yields $\mathrm{p} K_{\mathrm{a}}^{\mathrm{C}}=2.15$, indicating that $1 \mathrm{CEtH}^{2+}$ is only slightly more acidic than ITEtH $^{2+}$. This is a reasonable result.

\subsection{Laser flash spectroscopic investigation of the photodecoloration step}

The reaction sequence

$\mathrm{TEt}^{+} \stackrel{h \nu}{\longrightarrow} \mathrm{CEt}^{+} \longrightarrow \mathrm{SEt}^{+}$

can be kinetically resolved in detail when photoexciting $\mathrm{TEt}^{+}{ }^{+}$with a short (approximately $20 \mathrm{~ns}$ ) laser pulse. The transient absorption signals observed at some characteristic wavelengths are shown in Fig. 5 . At $\lambda>470 \mathrm{~nm}$, the signal consists of a single bleaching step ( $\mathrm{TEt}^{+} \rightarrow \mathrm{CEt}^{+}$) which is completed by the time the laser pulse is over. In the region $380>\lambda>470 \mathrm{~nm}$, the bleaching occurs in two steps, the first ending with the laser pulse and the second $\left(\mathrm{CEt}^{+} \rightarrow \mathrm{SEt}^{+}\right)$clearly continuing after the laser pulse. For this second step which obeys monoexponential kinetics, a rate constant of $1.9 \times 10^{7}$ $\mathrm{s}^{-1}$ is evaluated. At $\lambda=355 \mathrm{~nm}$, the fast step is not observed. Obviously this wavelength corresponds to an isosbestic point between $\mathrm{TEt}^{+}$and $\mathrm{CEt}^{+}$. In the region $320>\lambda>350$ $\mathrm{nm}$, the initial fast process corresponds to a transient increase in absorbance $\left(\epsilon_{\mathrm{CEt}^{+}}>\epsilon_{\mathrm{TEt}^{+}}\right)$. At $315 \mathrm{~nm}$, the second, slower step is not apparent in the transient signal because here we have the isosbestic point between $\mathrm{CEt}^{+}$and $\mathrm{SEt}^{+}$. Finally, at $\lambda<310 \mathrm{~nm}$, both absorption changes are positive. In this region $\epsilon_{\mathrm{TEt}+}<\epsilon_{\mathrm{CEt}^{+}}<\epsilon_{\mathrm{SEt}+}$.

The transient signals observed are fully consistent with the sequence of eqn. (9). No transient absorption of an electronically excited intermediate is detected. This 

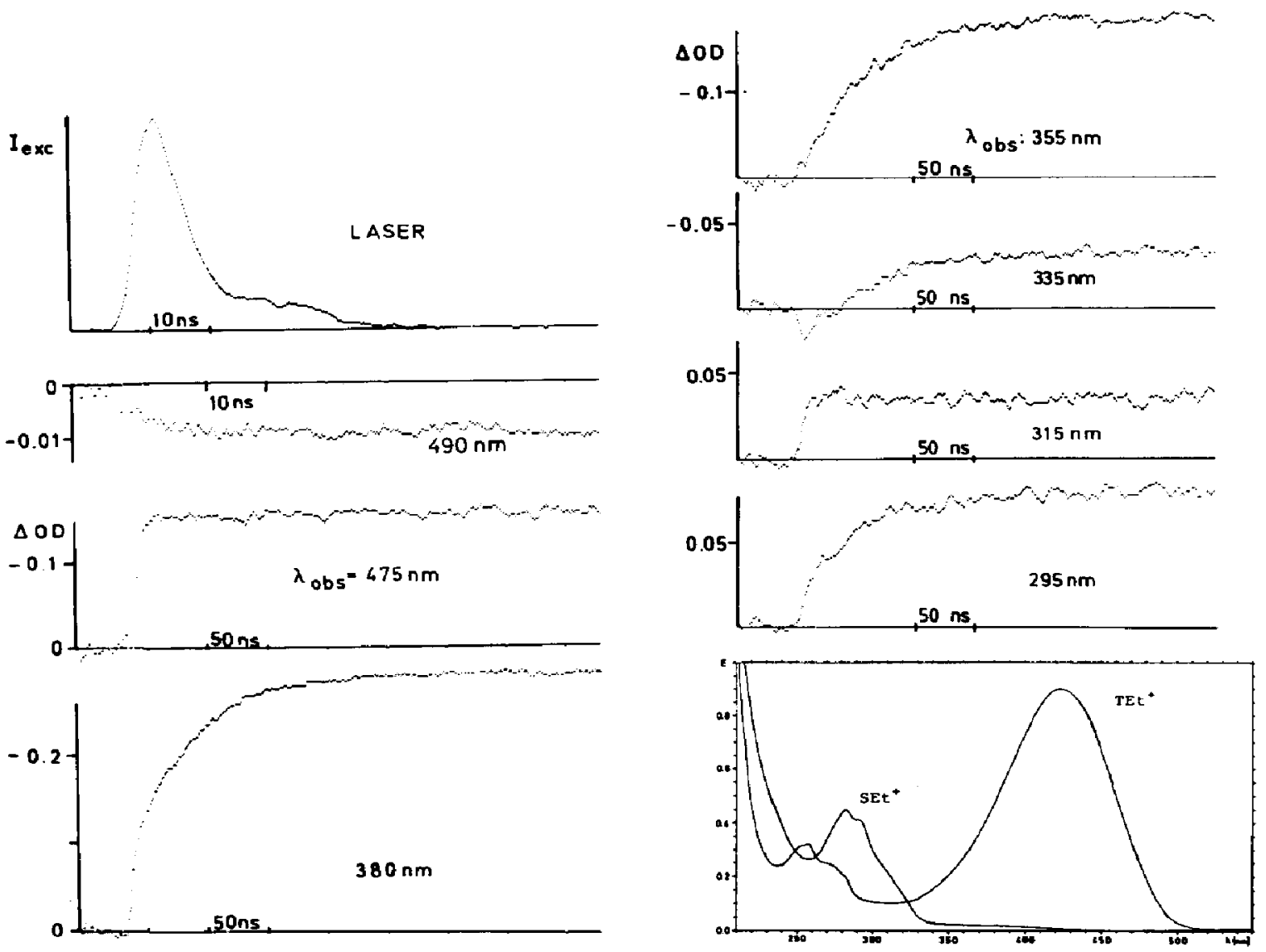

Fig. 5. Transient absorption signals observed on laser flash excitation at $428 \mathrm{~nm}$ of $1 \mathrm{TEt}^{+}$ $\left(c=4 \times 10^{-5} \mathrm{M}\right)$ in methanol. The time profile of the laser pulse, observed by stray light detection (arbitrary units), is also shown.

interpretation is corroborated by the observation that oxygen has no effect on the observed transient kinetics. The long-time absorption changes induced by laser flash excitation are identical with those observed in steady illumination experiments (see Fig. 6). This observation provides an excellent basis for an absolute scaling of the absorption changes during the course of the laser-induced kinetics.

Due to the finite duration of the laser pulse (see Fig. 5) and the short conversion time of $\mathrm{CEt}^{+} \rightarrow \mathrm{SEt}^{+}$, the transient absorption cannot be simply converted to the difference $\epsilon_{\mathrm{TEt}^{+}}-\epsilon_{\mathrm{CE}^{-}}$because the complete turnover may not be attained before the end of the laser pulse and/or because $\mathrm{CEt}^{+}$may already be partially converted into $\mathrm{SEt}^{+}$. A convolution procedure must be applied to determine the changes in the extinction coefficients $\Delta \epsilon_{\mathrm{C} / \mathrm{T}}=\left(\epsilon_{\mathrm{CEt}^{+}}-\epsilon_{T_{\mathrm{t}^{+}}}\right)$and $\Delta \epsilon_{\mathrm{S} / \mathrm{C}}=\left(\epsilon_{\mathrm{SEt}^{+}}-\epsilon_{\mathrm{CEt} t^{+}}\right)$for any wavelength of observation. This procedure is based on the following expression for the actual time dependence of the transient absorption change $\Delta A$

$\Delta A(t)=\phi_{\mathrm{tc}} \int_{0}^{t} I_{\mathrm{abs}}\left(t^{\prime}\right)\left[\Delta \epsilon_{\mathrm{C} / \mathrm{T}} \exp \left\{-k_{\mathrm{cs}}\left(t-t^{\prime}\right)\right\}+\Delta \epsilon_{\mathrm{s} / \mathrm{C}}\left(1-\exp \left\{-k_{\mathrm{cs}}\left(t-t^{\prime}\right)\right\}\right] \mathrm{d} t^{\prime}\right.$ 


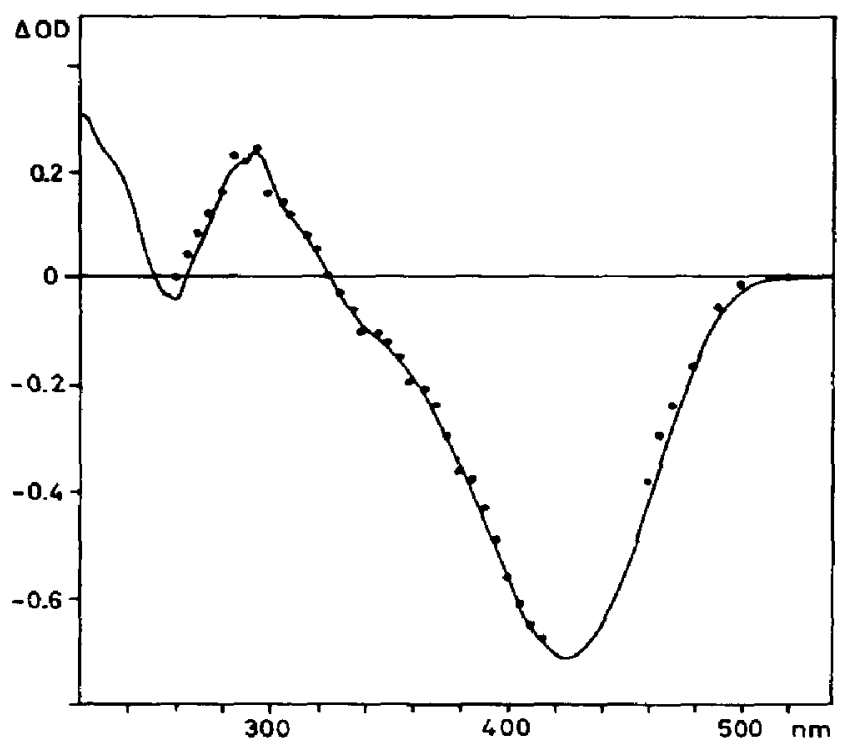

Fig. 6. Comparison of the photochemical absorption changes obtained for $1 \mathrm{TEt}^{+}$by continuous irradiation (full line) and laser photolysis (points). The laser results, corresponding to transient absorption changes recorded at $300 \mathrm{~ns}$ after the laser pulse, are normalized to fit the continuous illumination results at $400 \mathrm{~nm}$.

$$
=\phi_{\mathrm{tc}} \int_{0}^{t} I_{\mathrm{abs}}\left(t^{\prime}\right) \Delta \epsilon_{\mathrm{S} / \mathrm{C}} \mathrm{d} t^{\prime}+\phi_{\mathrm{tc}} \int_{0}^{t} I_{\mathrm{abs}}\left(t^{\prime}\right)\left(\Delta \epsilon_{\mathrm{c} / \mathrm{T}}-\Delta \epsilon_{\mathrm{S} / \mathrm{T}}\right) \exp \left\{-k_{\mathrm{cs}}\left(t-t^{\prime}\right)\right\} \mathrm{d} t^{\prime}
$$

Here $I_{\text {abs }}(t)$ is the photon concentration (number of einsteins per volume) absorbed by $\mathrm{TEt}^{+}$in the laser path through the cuvette. At times $t \gg k_{\mathrm{cs}}^{-1}$ this expression simplifies to

$\Delta A(\infty)=\phi_{\mathrm{tc}} \Delta \epsilon_{\mathrm{s} / \mathrm{C}} \int_{0}^{\infty} I_{\mathrm{abs}}\left(t^{\prime}\right) \mathrm{d} t^{\prime}$

The concentration of photons absorbed may be related to the relative intensity $L(t)$ of the laser light, measured as a stray light signal (see Fig. 5)

$$
I_{\mathrm{abs}}(t)=\frac{N_{\mathrm{I}} L(t)}{\int_{0}^{\infty} L\left(t^{\prime}\right) \mathrm{d} t^{\prime}} \frac{10^{E^{\prime}(\infty)}-1}{10^{E^{\prime}(0)}-1}=N_{\mathrm{I}} I_{\mathrm{abs}}{ }^{\prime}(t)
$$

Here $N_{I}$ is a normalization constant and $E^{\prime}(0)$ and $E^{\prime}(\infty)$ are the optical densities at the laser wavelength before and after the laser flash respectively. The latter expression takes into account the decreasing absorbance of the educt due to its bleaching during the laser pulse. Equation (12) is strictly valid only if the laser light is exclusively absorbed by the educt which, under the conditions of our experiments, holds true to a good approximation.

Comparing $\Delta A(\infty)$ with $\int_{0}^{\infty} I_{\mathrm{abs}}\left(t^{\prime}\right) \mathrm{d} t^{\prime}$ we obtain $N_{\mathrm{I}} \phi_{\mathrm{tc}} \Delta \epsilon_{\mathrm{S} / \mathrm{C}}$ from eqn. (11). The quantity $N_{\mathrm{I}} \phi_{\mathrm{tc}} \Delta \epsilon_{\mathrm{c} / \mathrm{r}}$ is obtained by fitting the numerically calculated integral (eqn. (10)) to the observed $\Delta A(t)$. Thus the ratio $\Delta \epsilon_{\mathrm{C} / \mathrm{T}} / \Delta \epsilon_{\mathrm{S} / \mathrm{C}}$ can be fixed. The absolute values 
are obtained by the equation

$\Delta \epsilon_{\mathrm{C} / \mathrm{T}}+\Delta \epsilon_{\mathrm{S} / \mathrm{C}}=\epsilon_{\mathrm{SEl}+}-\epsilon_{\mathrm{TLi}+}$

The difference between the extinction coefficients $\epsilon_{\mathrm{SEt}^{+}}-\epsilon_{\mathrm{TEt}^{+}}$is easily obtained from the continuous illumination experiments since $\mathrm{TEt}^{+}$and $\mathrm{SEt}^{+}$have non-overlapping spectral regions of absorption.

The complete spectrum thus evaluated for the intermediate species $\mathrm{CEt}^{+}$is shown in Fig. 7 together with those of $\mathrm{TEt}^{+}$and $\mathrm{SEt}^{+}$. As can be seen, the spectra of CEt ${ }^{+}$ and $\mathrm{TEt}^{+}$are related to each other in a similar manner to those of $\mathrm{CEtH}^{2+}$ and $\mathrm{TEtH}^{2+}$. A hypsochromic shift of about $40 \mathrm{~nm}$ occurs for both $\mathrm{CEt}^{+}$and $\mathrm{TEt}^{+}$when the dications are formed.

As has been mentioned, the kinetics of the process $\mathrm{CEt}^{+} \rightarrow \mathrm{SEt}^{+}$are exhibited uniformly throughout the spectrum. They were measured as a function of temperature and the activation parameters were obtained. These are collected in Table 1 and are compared with those of the other compounds.

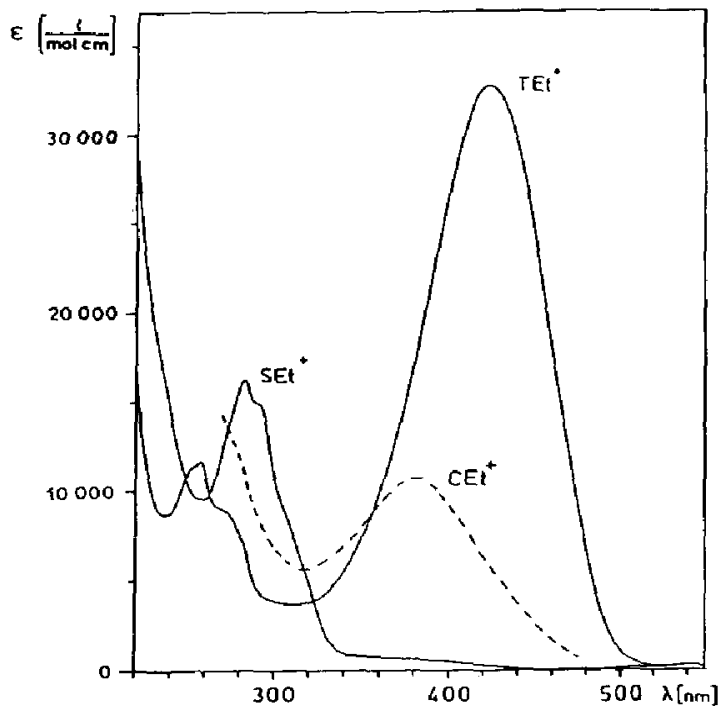

Fig. 7. Spectrum of intermediate $1 \mathrm{CEt}^{+}$, determined from the laser photolysis experiments, compared with those of $1 \mathrm{TEt}^{+}$and $1 \mathrm{SEt}^{+}$.

TABLE 1.

Characteristic protolytic, photochemical and kinetic parameters of the inversely photochromic compounds investigated in methanol

\begin{tabular}{llllll}
\hline Parameter & 1TEt $^{+}$ & $2 \mathrm{TH}^{+}$ & $3 \mathrm{TH}^{+}$ & $4 \mathrm{TH}^{+}$ & $\mathbf{5 T H}^{+}$ \\
\hline $\mathrm{p} K_{\mathrm{a}}\left(\mathrm{TH}_{2}{ }^{2+}\right)$ & 2.6 & 2.9 & 3.1 & 3.2 & 1.3 \\
$\phi_{\mathrm{ct}}$ & 0.54 & 0.55 & 0.52 & 0.45 & 0.56 \\
$k_{\mathrm{cs}}\left(\mathrm{s}^{-1}\right)(300 \mathrm{~K})$ & $2.2 \times 10^{7}$ & $1.8 \times 10^{7}$ & $1.1 \times 10^{7}$ & $1.2 \times 10^{6}$ & $8.0 \times 10^{6}$ \\
$E_{\mathrm{a}}\left(\mathrm{kJ} \mathrm{mol}^{-1}\right)$ & 28.5 & 30.2 & 32.3 & 37.7 & 30.2 \\
$A\left(\mathrm{~s}^{-1}\right)$ & $2.1 \times 10^{12}$ & $3.3 \times 10^{12}$ & $4.0 \times 10^{12}$ & $4.0 \times 10^{12}$ & $1.3 \times 10^{12}$ \\
\hline
\end{tabular}




\subsection{Investigation of spiro compounds $2 S$ to $5 S$}

With the results for compound $\mathrm{ISEt}^{+}$as a reference, clearly interpretable kinetic results can also be obtained with the protonated compounds $2-5 \mathrm{SH}^{+}$. Thus for $2 \mathrm{~S}$ the protolytic equilibrium

$2 \mathrm{~S}+\mathrm{H}^{+} \rightleftarrows 2 \mathrm{SH}^{+}$

has a $p K_{a}^{S}$ value of 4.5 (see Fig. 8). The weak long-wavelength absorption indicates that here, as in the case of $1 \mathrm{SEt}^{+}$, some small fraction of $2 \mathrm{CH}^{+}$exists in equilibrium with $2 \mathrm{SH}^{+}$. On increasing the proton concentration, the equilibrium

$\left(2 \mathrm{SH}^{+} \rightleftarrows 2 \mathrm{CH}^{+}\right) \stackrel{\mathrm{H}^{+}}{\rightleftarrows} 2 \mathrm{CH}_{2}^{2+}$

is shifted (see Fig. 9) with an effective $\mathrm{p} K_{\mathrm{a}}\left(2 \mathrm{CH}_{2}{ }^{2+} \rightarrow 2 \mathrm{SH}^{+}+\mathrm{H}^{+}\right.$) value of 1.2 (compare with 1.1 for $1 \mathrm{SEtH}^{2+}$ ). At room temperature, thermal conversion of $2 \mathrm{CH}_{2}{ }^{2+}$ to $2 \mathrm{TH}_{2}{ }^{2+}$ occurs with a rate constant $k_{\mathrm{ct}}^{\prime}$ of $3 \times 10^{-4} \mathrm{~s}^{-1}$ (compare with $3.4 \times 10^{-4} \mathrm{~s}^{-1}$ for $1 \mathrm{CEtH}^{2+}$ ), whereas for $2 \mathrm{SH}^{+}$the rate constant of thermocoloration is $7 \times 10^{-6} \mathrm{~s}^{-1}$ (compare with $8.3 \times 10^{-6} \mathrm{~s}^{-1}$ for $1 \mathrm{SEt}^{+}$).
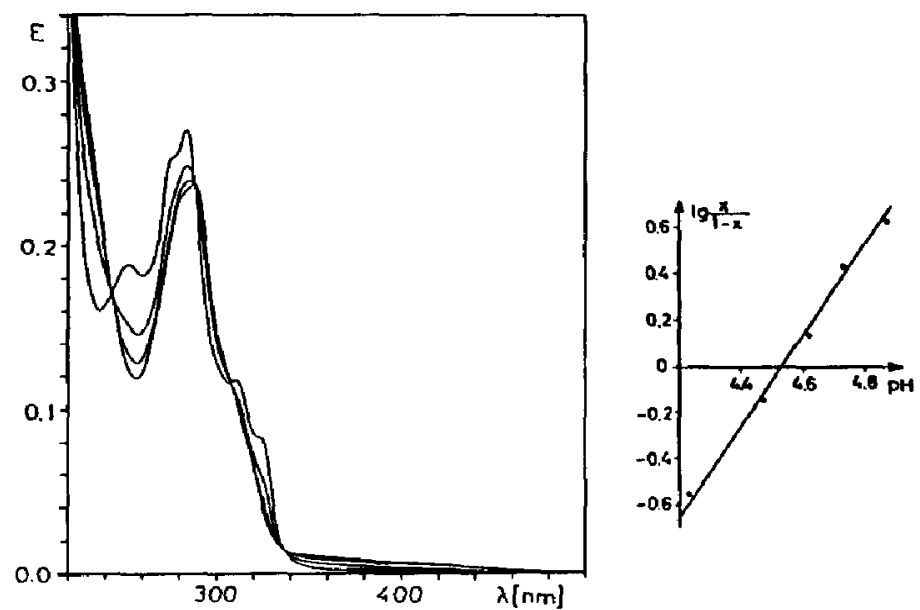

Fig. 8. Spectral absorbance change due to a shift in the equilibrium $2 \mathrm{~S}-2 \mathrm{SH}^{+}$with $\mathrm{pH}$ and determination of $\mathrm{p} K_{a}$ in methanol. The sample concentration was $1.7 \times 10^{-5} \mathrm{M}$.
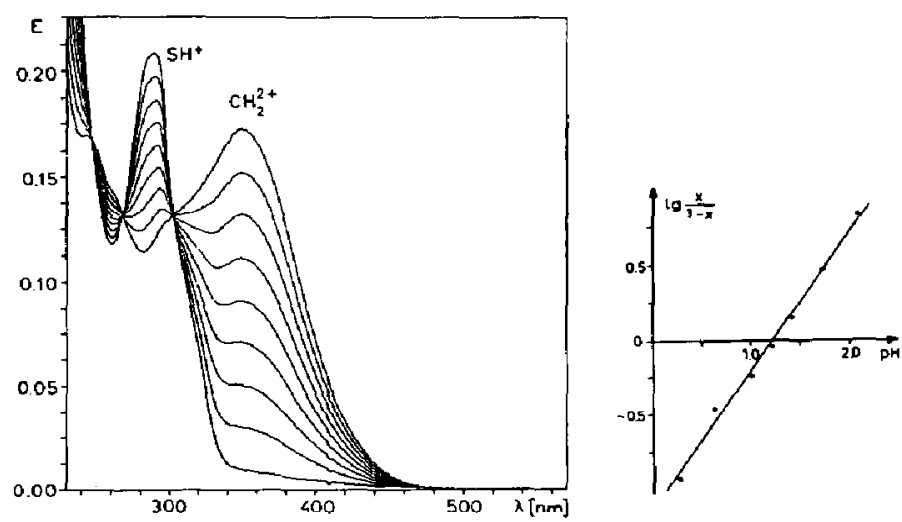

Fig. 9. Spectral absorbance change due to a shift in the equilibrium $2 \mathrm{SH}^{+}-2 \mathrm{CH}_{2}{ }^{2-}$ and determination of $\mathrm{p} K_{\mathrm{a}}$ in methanol. The sample concentration was $1.5 \times 10^{-5} \mathrm{M}$. 
Stationary illumination of $2 \mathrm{TH}^{+}$gives a quantum yield of photodecoloration of 0.55 (compare with 0.54 for $1 \mathrm{TEt}^{+}$). Furthermore the results obtained by laser flash spectroscopy are also very similar to those of $1 \mathrm{TEt}^{+}$.

Characteristic data pertaining to the photochemical decoloration of the $n \mathrm{TH}^{+}$ forms and the thermal ring closure $\left(k_{\mathrm{cs}}\right)$ are listed in Table 1. The spectra obtained for the intermediate forms $3 \mathrm{CH}^{+}$and $4 \mathrm{CH}^{+}$are presented in Figs. 10 and 11.

From the data in Table 1, it can be seen that the five compounds behave very similarly. The introduction of substituents in the indolino and imidazodihydroquinoline parts of the molecule causes only moderate changes in $\mathrm{p} K_{\mathrm{a}}$ values, quantum yields and rate constants. At room temperature, the rate constants of ring closure of the cis conformers are of the order of $10^{7} \mathrm{~s}^{-1}$ which, to our knowledge, represents the

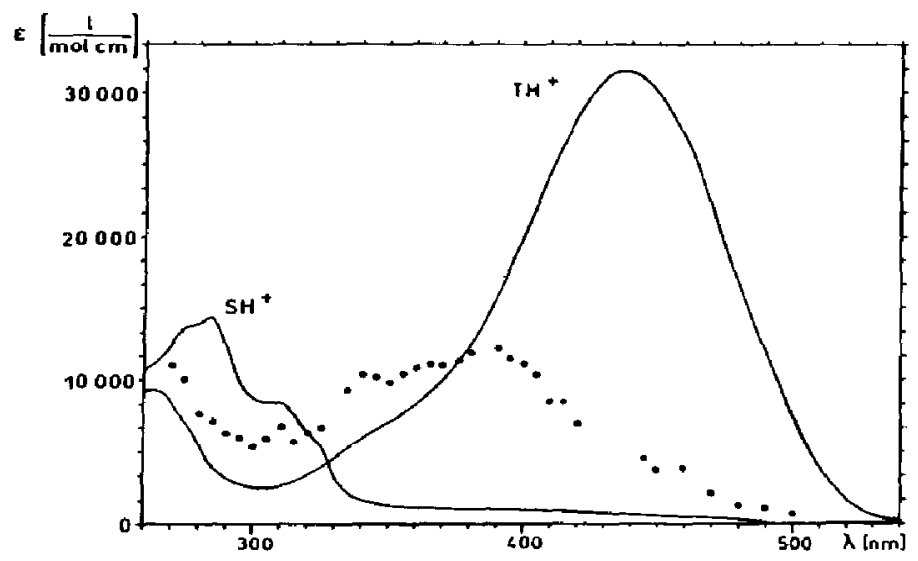

Fig. 10. Spectrum of intermediate $3 \mathrm{CH}^{+}$, determined from the laser photolysis experiments, compared with those of $3 \mathrm{TH}^{+}$and $3 \mathrm{SH}^{+}$.

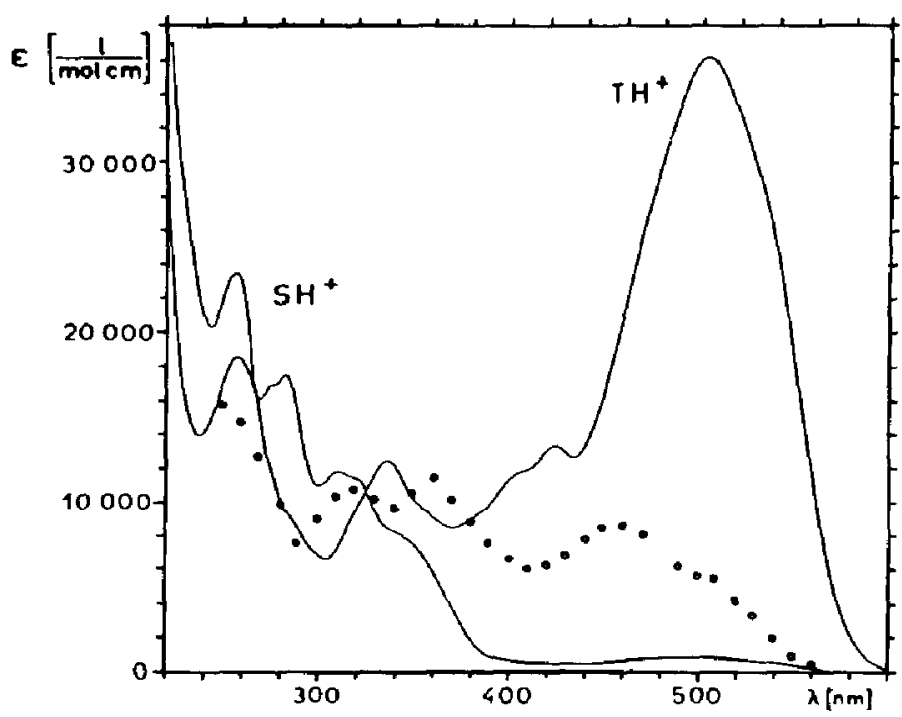

Fig. 11. Spectrum of intermediate $4 \mathrm{CH}^{+}$, determined from the laser photolysis experiments, compared with those of $4 \mathrm{TH}^{+}$and $4 \mathrm{SH}^{+}$. 
fastest thermal electrocyclic formation of a six-membered ring observed so far. For example, thermal cyclizations of the merocyanines forming indolinospiropyrans occur with rate constants in the range $10^{-5}-10^{3} \mathrm{~s}^{-1}$ [6]. The essential difference from the present cases is that in our compounds, the activation barrier of isomerization between the cis form and the thermodynamically more stable trans conformer of the openchain conjugated system is higher than for the merocyanines. Hence, after the photoinduced conformational change $\mathrm{TH}^{+} \rightarrow \mathrm{CH}^{+}$, the system is trapped in a conformation which is sterically favourable for the ring-closure step. Its rate constant $\left(k_{\mathrm{cs}}\right)$ is directly measured in our experiment. The conformational change associated with the activation step of ring closure in our experiments cannot be drastic since the "normal" frequency factors are several orders of magnitude higher than expected for six-ring formation from a conformationally unrestricted open chain. In the thermal decoloration of merocyanines leading to the spiropyrans, the trans conformer dominates, and the effective rate constant measured for decoloration typically corresponds to the ringclosure rate constant of the cis conformer multiplied by the equilibrium fraction of cis conformers.

Although, after the photochemical trans-cis isomerization of our open-chain chromophore the olefinic double bond is in the appropriate configuration for successive ring closure, there may still be other internal rotations involved before spiro-bond formation can take place. A detailed study of the viscosity and temperature dependence of the (photoinduced) thermal ring-closure kinetics of $1 \mathrm{TEt}^{+}$[22], has revealed that the fast rate process $\left(10^{7} \mathrm{~s}^{-1}\right)$ observed after photochemical trans-cis isomerization of $1 \mathrm{TEt}^{+}$is mainly due to a rotational rearrangement (see Fig. 12). The ring closure proper, i.e. from the conformational minimum of $\mathrm{CEt}^{+}$which is closest to the SEt ${ }^{+}$ configuration, occurs with a rate constant of $6 \times 10^{7} \mathrm{~s}^{-1}$ at room temperature [22]. The rate constants reported in Table 1 are characteristic of the low viscosity limit
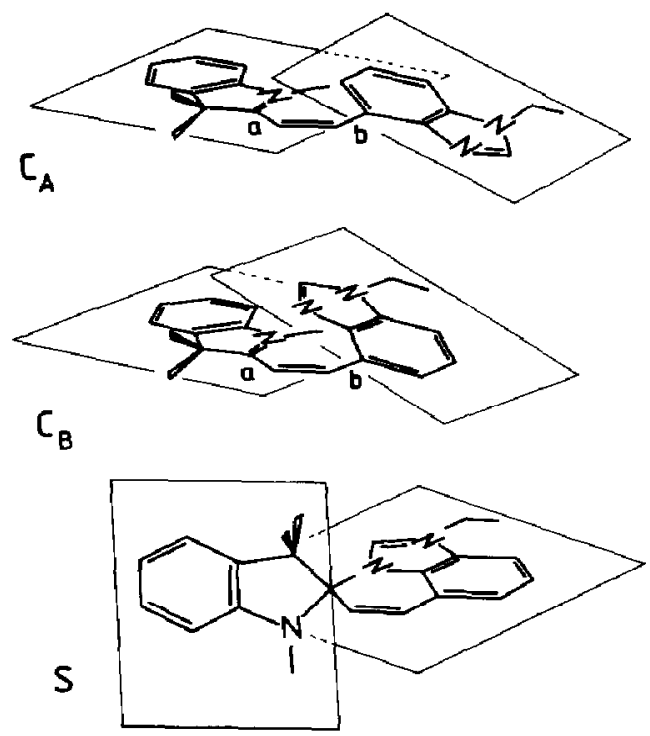

Fig. 12. At low viscosities, rotamers $C_{A}$ and $C_{B}$ are in fast equilibrium after photochemical isomerization of the trans isomer. Due to a preponderance of rotamer $C_{A}$, the effective rate constant $k_{\mathrm{cs}}$ for the process $\mathrm{C} \rightarrow \mathrm{S}$ is significantly smaller than the rate constant of the ringclosure step $C_{B} \rightarrow S$. 
and correspond to the product of an equilibrium constant between cis rotamers $C_{A}$ and $\mathrm{C}_{\mathrm{B}}$ and the "proper" ring closure rate constant $k\left(\mathrm{C}_{\mathrm{B}} \rightarrow \mathrm{S}\right)$.

\section{Conclusions}

We have spectroscopically identified the short-lived (ground state) cis conformers $\mathrm{CH}^{+}$as distinct intermediates in the photodecoloration of the inversely photochromic forms $\mathrm{TH}^{+}$of compounds 1-5. Thus the photochemical step in this photodecoloration comesponds to a trans-cis isomerization and is followed by a thermal ring-closure reaction. The latter process occurs with a rate constant of typically $10^{7} \mathrm{~s}^{-1}$ at room temperature and with an activation energy of the order of $30 \mathrm{~kJ} \mathrm{~mol}^{-1}$; the thermal reversion of $\mathrm{CH}^{+}$to $\mathrm{TH}^{+}$is more than ten orders of magnitude slower than the ringclosure reaction. The ring opening $\mathrm{SH}^{+} \rightarrow \mathrm{CH}^{+}$is only 50-100 times slower than ring closure, meaning that the thermodynamic bias between $\mathrm{SH}^{+}$and $\mathrm{CH}^{+}$is at an optimum with respect to the maximization of the rate of thermal ring opening and the equilibrium ratio of $\mathrm{SH}^{+}$to $\mathrm{CH}^{+}$.

\section{Acknowledgment}

D. K. and U. E. S. gratefully acknowledge support of this investigation by the Deutsche Forschungsgemeinschaft.

\section{References}

1 R. Dessauer and J. P. Paris, Adv. Photochem., I (1963) 275.

2 E. Fischer, Fortschr. Chem. Forsch., 7 (1967) 605.

3 G. H. Brown (ed.), Techniques of Chemisty, Vol. 3, Photochromism, Wiley, New York, 1971.

4 H. Dürr and H. Bouas-Laurent (eds.), Photochromism, Elsevier, Amsterdam, 1990.

5 R. C. Bertelson, in G. H. Brown (ed.), Techniques of Chemistry, Vol. 3, Photochromism, Wiley, New York, 1971, p. 45.

6 R. Gugliemetti, in H. Dürr and H. Bouas-Laurent (eds.), Photochromism, Elsevier, Amsterdam, 1990 , p. 314.

7. R. Heiligman-Rim, Y. Hirshberg and E. Fischer, J. Phys. Chem., 66 (1962) 2465, 2470.

8 T. Bercovici, R. Heiligman-Rim and E. Fischer, Mol. Photochem., 1 (1969) 23.

9 G. L. Lashkov and A. V. Shablya, Opt. Spectrosc. (U.S.S.R.), 19 (1965) 821.

10 A. S. Kholmanskii and N. I. Kunavin, Russ. J. Phys. Chem., 58 (1984) 602.

11 M. Gehrtz, C. Bräuchle and J. Voitländer, J. Am. Chem. Soc., 104 (1982) 2094.

12 H. Balli and A. Keller, Chimia, 27 (1973) 646.

13 H. Balli, Chimia, 31 (1977) 336.

14 A. Keller, Doctoral Thesis, Universität Basel, 1972.

15 R. Konstanzer, Doctoral Thesis, Universität Basel, 1977.

16 G. Gauglitz, J. Photochem., 5 (1976) 41.

G. Gauglitz and S. Hubig, J. Photochem., 15 (1981) 255.

17 T. Ulrich, U. E. Steiner and W. Schlenker, Tetrahedron, 42 (1986) 6131.

18 T. Ulrich, Doctoral Thesis, Universität Konstanz, 1986.

19 H. G. O. Becker (ed.), Einfühning in die Photochemie, Georg Thieme Verlag, Stuttgart, 1983.

20 D. Gegiou, K. A. Muszkat and E. Fischer, J. Am. Chem. Soc., 94 (1968) 9142.

21 G. Bartocci, P. Bortolus and Mazzucato, J. Phys. Chem., 77 (1973) 605.

22 D. Kühn and U. E. Steiner, to be published.

D. Kühn, Doctoral Thesis, Universität Stuttgart, 1987. 